Article

\title{
A Study on the Influence of Latin Dance and Tai Chi Exercise on Balance and Knee Joint Proprioception
}

\author{
Huihui Wang, Zhongqiu Ji *, Guiping Jiang, Xibian Jiao and Weitong Liu \\ College of P.E and Sports, Beijing Normal University, Beijing 100875, China; 15101579368@163.com (H.W.); \\ jiang_guiping0401@126.com (G.J.); 369569000@qq.com (X.J.); 497208486@qq.com (W.L.) \\ * Correspondence: jizhongqiu61@bnu.edu.cn
}

\begin{abstract}
Objective: To study the influence of Latin dance and Tai Chi on knee joint proprioception and balance. Method: All experiments were performed in the Beijing Normal University School of Physical Education and Sport Biomechanics Laboratory. An isokinetic test system (Biodex system 4, BS4) and a balance testing system (Biodex Balance System, BBS)from the United States Biodex medical system were used to test related indexes. Results: In the LOS test, the overall score of Latin group is much better than sedentary group. In the ASL test, Latin group have a better balance ability in anterior and posterior direction and overall score than sedentary group $(\mathrm{p}<0.05)$; TAI CHI group have a better balance ability than sedentary group in anterior and posterior direction $(\mathrm{p}<0.05)$ and right and left direction $(\mathrm{p}<0.01)$. When the knee joint was at 15,30 , and 45 degree positions, the Tai Chi exercise group was much better than sedentary group $(\mathrm{P}<0.05)$, and the 45 degree position in the Tai Chi group was much better than that in the Latin dance group $(\mathrm{P}<0.01)$. When the knee joint was at the 30 degree position, the quadriceps force sense was significantly better in the Tai Chi group than in the Latin dance and sedentary groups. In a test of the four angles, the hamstring force sense in the Tai Chi exercise group was obviously better than that in the sedentary group $(\mathrm{P}<0.05)$; at the 15 degree position, the Tai Chi group was significantly better than the Latin group $(\mathrm{P}<0.05)$; and at 30 degree position, the hamstring force sense of the Latin group was obviously better than that of the sedentary group $(\mathrm{P}<0.05)$.
\end{abstract}

Keywords: latin dance; tai chi; knee proprioception

\section{Introduction}

Latin dance, which originated in group dance events in Latin America, includes the rumba, samba, Cha Cha Cha, bullfighting dances and cowboy dances ${ }^{[1]}$. Latin dance steps are flexible and changeable. With the rhythm of music, the dancer must show their beauty of posture through speed, strength and flexibility ${ }^{[2]}$. When performing, the dancers must have good posture control and balance to complete technical actions of different difficulties to obtain a more beautiful posture and performance ${ }^{[3,4]}$. When practicing Tai Chi, practitioners must make repeated body shifts, rotate and alternate single foot support. All of these actions require precise joint control, muscle coordination, and good body balance ${ }^{[5]}$. These two different forms of exercise can improve body balance, and proprioception capability[6]. Balance, as an essential function in human daily life and sports activities, can be defined as central nervous system input from proprioceptive, vestibular, and visual afferent nerve signals, with analysis of signal integration. Human body posture is controlled by motor neurons that regulate muscle activity ${ }^{[7,8]}$. Proprioception in the central nervous system accept mechanical nerve impulses from joints, ligaments, tendons, joint capsules, muscles, and skin receptors to eventually lead to corresponding reflex and motion control[8]. Balance ability testing includes many components, such as static postural stability (postural stability, PS), dynamic posture stability limits (limits of stability, LOS), and dynamic fall risk (dynamic fall index, DFI). Proprioception testing methods mainly include motion perception, position perception, and force sense tests ${ }^{[-11]}$. Studies of TaiChi proprioception have focused mainly on older people and on ankle or knee proprioception. In this paper, the influence of two types of training methods on 
proprioception and balance were analysed using an An isokinetic muscle testing system and A balance testing system.

\section{Subjects and Methods}

\subsection{Subjects}

All subjects were from Beijing Normal University. For the Latin group, 11 Subjects were recruited, and they all practiced at least 1 time a week and had an average training period of $5.06+$ 2.35 years. Tai Chi group included 8 girls who had Tai Chi class 2 times a week for 90 minutes each session and at least 18 weeks of exercise time. A sedentary group of 8 girls was included. All subjects voluntarily participated in this experiment, were in good health, and had no disease or knee injuries. Basic information about the subjects is shown in Table 1.

Table1. basic information for subjects in the Latin, tai chi and control groups

\begin{tabular}{ccccc}
\hline Group & Num(subjects) & Age (years) & Height $(\mathrm{cm})$ & Weight $(\mathrm{Kg})$ \\
\hline TaiChi & 8 & $20.50 \pm 1.41$ & $160.63 \pm 5.63$ & $50.00 \pm 5.37$ \\
Latin & 11 & $22.64 \pm 3.56$ & $166.00 \pm 5.42$ & $57.55 \pm 6.65$ \\
Sedentary & 8 & $22.33 \pm 1.41$ & $162.67 \pm 4.62$ & $50.00 \pm 6.21$ \\
\hline
\end{tabular}

\subsection{Methods}

An isokinetic muscle testing system (Biodex System 4, BS4) and A balance testing system (Biodex Balance System, BBS; Biodex Medical Systems, USA) were used for measurement.The BS4 system test include passive position sense(PS) and force sense (FS).

\subsubsection{BBS test}

The BBS system includes an angle sensor and foot position detection platform, for Athlete Single Leg (ASL) test and Limits of Stability (LOS) test. Before the actual test, subjects stand on the platform, to become familiar with the testing process. ASL test: The test platform was set at static level, with the subject using the right leg to support, placing hands naturally on the sides, and moving the right foot to the center on the screen before subject. The subject was instructed to maintain, with eyes closed, the centre of gravity, for a test time of $20 \mathrm{~s}$. The lower the score, the better the ability to control posture. LOS test: With the horizontal platform set at static level, the subjects stood with both feet on the platform; when the body centre of gravity and bulls eye coincided, the foot position and angle were recorded. With the directions displayed at random, the subjects were asked to complete the directional tasks displayed on a screen as quickly as possible. A total of 3 tests were performed, at intervals of $5 \mathrm{~s}$, with the third test used for results. The test results were used to determine a total score (LOS-Overall) and test completion time (LOS-Time). The higher the total score and shorter the completion time, the better the ability of the subjects to transfer the centre of gravity ${ }^{[2]}$.

\subsubsection{BS4 test}

With subjects seated on the Biodex device, and the angle between the back and seat maintained at 90 degrees, the right leg was fixed on the power arm, and the thigh was attached to the seat. Before starting the actual test, the subjects perform warm-up exercises in passive isokinetic mode at a knee angular velocity of 60 degree/s, 30 times.

PS test: The knee joint of the subjects was passively set to 15, 30, and 45 degrees from 90 degrees(definition: the knee flexion position was 90 degrees, and extension was 0 degrees.). The knee joint was held for $5 \mathrm{~s}$ to allow the participants to feel the position of the knee joint in the relaxed state. When subjects were passive to the 30,45 and 60 degree positions and pressed a button, The dynamic head brought the knee of the subjects from flexion to extension. Repeated tests were carried out 3 times at each angle, and the angular velocity of the passive motion was 5 degrees/s. The absolute errors between the target value and the test value were the test values[18]. FS test: The torso and 
dominant thigh were fixed to the test chair to remove the compensation from other limbs with the centre of knee rotation and the dynamometer on a straight line. Test patterns for the isometric strength test and the maximum voluntary isometric contraction (Maximal voluntary isometric contraction, MVIC) were tested at the 15, 30, and 45 degrees positions. Maximum isometric Quadriceps and Hamstring muscle strength were tested in each knee joint angle with a test time of 5 $\mathrm{s}$, and the tests were repeated 3 times, with rest periods of $5 \mathrm{~s}$ between each test and with $300 \mathrm{~s}$ of rest between test sets. The coefficient of variation (coefficient of variance, CV) required control less than $15 \%$. After 30 minutes of rest, using 50\% MVIC as the target force value, the strength of the knee joint was tested using visual feedback of the target force on the computer screen. Subjects were asked to close their eyes and use the quadriceps/hamstrings of their knee joint to reproduce the target force value, and the measured value was recorded. The peak torque (PT) of the quadriceps/hamstrings, the antagonistic ratio $(\mathrm{Q} / \mathrm{H})$ and absolute error between the difference force target values and the measured forces were recorded. The test was performed twice ${ }^{[13]}$.

\subsubsection{Statistical methods}

SPSS 13.0 statistical software was used for analysis of the test parameters, an independent sample $\mathrm{T}$ test were conducted between groups. All test indexes were expressed as mean \pm standard deviation; $\mathrm{P}<0.05$ indicates that the difference is statistically significant.

\section{Result}

\subsection{The result of Athlete Single Leg (ASL) test and Limits of Stability (LOS) test}

As shown in the Table 2 and Figure 1, in the LOS test, the overall score of Latin group is much better than sedentary group. In the ASL test, Latin group have a better balance ability in anterior and posterior direction and overall score than sedentary group $(\mathrm{p}<0.05)$; TAI CHI group have a better balance ability than sedentary group in anterior and posterior direction $(\mathrm{p}<0.05)$ and right and left direction $(\mathrm{p}<0.01)$.

Table2. Result for LOS test

\begin{tabular}{ccc}
\hline & Time(s) & Overall(points) \\
\hline Tai Chi & $36.50 \pm 5.90$ & $40.94 \pm 9.78$ \\
Latin & $35.00 \pm 5.20$ & $46.89 \pm 7.03$ \\
Sedentary & $43.00 \pm 13.67$ & $38.25 \pm 8.35 \#$ \\
\hline
\end{tabular}

Note: \# indicates a significant difference between the Latin dance and sedentary groups $(\mathrm{P}<0.05)$.
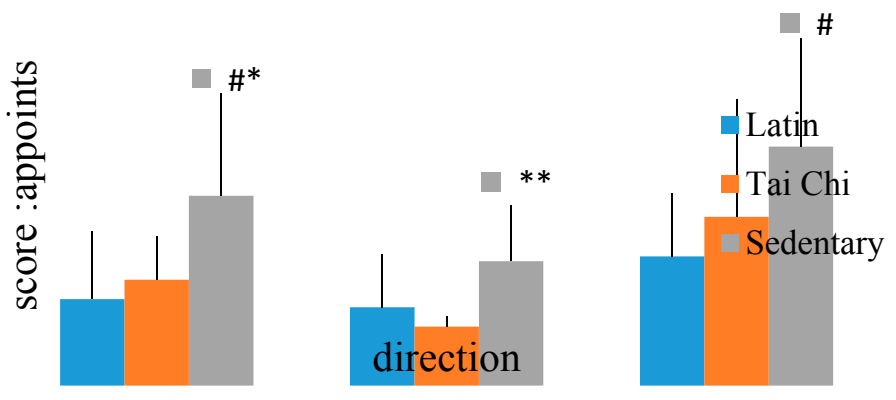

Figure 1. Result for ASL test

Note: * indicates the Tai Chi group had a significant difference from the Latin group and sedentary control group $(\mathrm{P}<0.05)$, *

* means that the Tai Chi group had a very significant difference from the Latin group and sedentary control group $(\mathrm{P}<0.01)$, \# indicates a significant difference between the Latin dance and sedentary groups $(\mathrm{P}<0.05)$ (the same below) .

\subsection{Analysis of knee position sense}


As shown in Table 3, when the knee was located at $45^{\circ}$, the absolute position angle was lower for the Tai Chi group than for the Latin dance group, and at the $15^{\circ}$ position, the results for the Tai Chi group were significantly lower than those of the control group $(\mathrm{P}<0.01)$. At $30^{\circ}$ and $45^{\circ}$, the Tai Chi group results were significantly lower than the control group results $(\mathrm{P}<0.05)$. Comparing the position of sleep between the Latin group and control group, there was no significant difference.

Table 3. Comparative analysis of different joint angles in the Latin, Tai Chi and Sedentary groups

\begin{tabular}{llll}
\hline \multirow{2}{*}{ Group } & \multicolumn{4}{l}{ Different joint angles (degree) } \\
\cline { 2 - 4 } & $15^{\circ}$ & $30^{\circ}$ & $45^{\circ}$ \\
\hline Tai Chi & $1.96 \pm 0.74$ & $2.55 \pm 1.65$ & $2.06 \pm 1.07$ \\
Latin & $2.83 \pm 1.64$ & $3.18 \pm 2.02$ & $4.06 \pm 1.64^{* *}$ \\
Sedentary & $3.78 \pm 1.49^{* *}$ & $4.53 \pm 1.74^{*}$ & $3.72 \pm 1.85^{*}$ \\
\hline
\end{tabular}

\subsection{Quadriceps force sense}

As shown in Table 4, when the knee was at the 30-degree position, the value of quadriceps force sense in the Tai Chi Group was significantly lower than that in the Latin dance $(\mathrm{P}<0.05)$ and sedentary groups $(\mathrm{P}<0.01)$. There was no significant difference in the force sense of the three groups when the knee joint positions were 15 and 45 degrees.

Table 4. Comparative analysis of different joint angles in the Latin dance group, Tai Chi group and sedentary group

\begin{tabular}{llll}
\multirow{2}{*}{ Group } & \multicolumn{3}{c}{ Different joint angles $($ degree $)$} \\
\cline { 2 - 4 } & $15^{\circ}$ & $30^{\circ}$ & $45^{\circ}$ \\
\hline Tai Chi & $2.66 \pm 1.68$ & $1.75 \pm 0.81$ & $2.63 \pm 2.71$ \\
Latin & $2.60 \pm 1.77$ & $4.04 \pm 1.98^{*}$ & $3.79 \pm 2.75$ \\
Sedentary & $2.65 \pm 1.04$ & $4.88 \pm 1.57^{* *}$ & $4.46 \pm 2.56$ \\
\hline
\end{tabular}

\subsection{Hamstrings force sense}

As shown in Table 5, there were significant differences between the Tai Chi and sedentary group sat 15,30 , and 45 degrees $(\mathrm{P}<0.05)$. There were significant differences between the Tai Chi and Latin groups $(\mathrm{P}<0.05)$ at 30 and degrees as well as significant differences between the Latin dance and sedentary groups. When the knee was at the 30-degree flexion position, the extensor strength absolute sense value of the Tai Chi Group was significantly lower than those of the Latin dance $(P<0.05)$ and control groups $(\mathrm{P}<0.01)$. There was no significant difference in the absolute value of the three groups at the 15-degree position.

Table 5. Comparative analysis of different joint angles in the Latin dance, Tai Chi and sedentary groups

\begin{tabular}{llll}
\hline \multirow{2}{*}{ Group } & \multicolumn{3}{l}{ Different joint angles (degree) } \\
\cline { 2 - 4 } & $15^{\circ}$ & $30^{\circ}$ & $45^{\circ}$ \\
\hline Tai Chi & $1.14 \pm 0.89$ & $2.31 \pm 1.71$ & $1.64 \pm 0.97$ \\
Latin & $2.25 \pm 1.35^{*}$ & $2.48 \pm 1.77$ & $2.10 \pm 1.09$ \\
Sedentary & $2.60 \pm 1.14^{*}$ & $5.58 \pm 4.08^{*} \#$ & $3.00 \pm 1.24^{*}$ \\
\hline
\end{tabular}

Note: * indicates the Tai Chi group had a significant difference from the Latin group and sedentary control group (P<0.05), * * means that the Tai Chi group had a very significant difference from the Latin group and sedentary control group(P $<0.01)$, \# indicates a significant difference between the Latin dance and sedentary groups $(\mathrm{P}<0.05)$.

\section{Discussion}

Comparing balance ability among Latin, TAI CHI and sedentary group, the score of Latin group was obviously higher than sedentary group, which explained that Latin dance can better improve the 
speed of shifting weight, and posture control in anterior and posterior direction. TAI CHI exercise can improve posture control in anterior/posterior and left/right direction. After comparing passive position sense and force sense among the Tai Chi, Latin and sedentary groups, we found that there were significant differences in passive position sense between the Tai Chi and sedentary groups.

The role of proprioceptors differs at different angles of the knee joints. In this study, when the knee joint is at 15 degrees, the Tai Chi Group passive position sense and hamstring force sense were significantly better than the sedentary group. At the same time, the Tai Chi group force sense was significantly better compared to the Latin dance group. This outcome shows that the practice of Tai Chi can, to a certain extent, better prevent injury in sports and falls in the elderly. Along with changes in the angle of the joint, the degree of relaxation of the knee joint around the ligament and medial ligament is different. Knee anterior cruciate ligament proprioception between 30 and 40 degree position played a major role. At the 30 degree position, the Tai Chi group passive position sense and force sense were significantly better than those of the sedentary group. At the same time, position sense was significantly better in the Tai Chi group compared to the Latin dance group. Additionally, the Latin flexor was significantly better in the no exercise group.

In the middle position of the knee joint movement at 45 degrees, the Tai Chi Group passive position sense was significantly better compared to the Latin dance and sedentary groups. The hamstring force sense of the Tai Chi group sense was also significantly better than that of the sedentary group. At the 60 degree position, there was no significant difference in passive position between the three groups, but the quadriceps and hamstring force sense for the Tai Chi group were significantly better than those of the Latin dance and sedentary groups. The hamstring force sense of the Tai Chi group was significantly better than that of the sedentary group, and the Latin dance group was much better than the no exercise group.

From the above analysis, we know that the influence of Tai Chi exercise on position sense is mainly reflected at the 15, 30 and 45 degree positions and that the main role of the muscle spindle angle occurs at 45 degrees. Latin dance practice affected only the force sense of the hamstring. Tai Chi exercise can improve proprioceptive ability in different aspects, improve the overall control of the knee joint and ultimately improve body balance. Latin dance exercises can improve body balance by improving the proprioception of force sense.

\section{Conclusion}

(1) In the LOS test, the overall score of Latin group is much better than sedentary group. In the ASL test, Latin group have a better balance ability in anterior and posterior direction and overall score than sedentary group $(\mathrm{p}<0.05)$; TAI CHI group have a better balance ability than sedentary group in anterior and posterior direction $(\mathrm{p}<0.05)$ and right and left direction $(\mathrm{p}<0.01)$.

(2) When the knee joint was at 15, 30, and 45 degree positions, Tai Chi exercise was much better than the control group $(\mathrm{P}<0.05)$. At the 45 degree position, the results for the Tai Chi group were much better than those for the Latin dance group $(\mathrm{P}<0.01)$.

(3) When the knee joint was at the 30 degree positions, the quadriceps force sense was significantly better in the Tai Chi group than in the Latin dance and sedentary control groups.

(4) In the test of four angles, the hamstring force sense in the Tai Chi exercise group was obviously better than the control group $(\mathrm{P}<0.05)$. At the 15 degree position, the results were significantly better compared to the Latin group $(\mathrm{P}<0.05)$. Finally, at 30 degree, the hamstring force sense of the Latin dance group was obviously better than that of the control group $(\mathrm{P}<0.05)$.

Acknowledgments: The National Social Science Foundation for the education of general subjects, an educational intervention research for promoting and influencing the development of children's action (BLA150063).

\section{References}

[1] Marquez DX, Wilbur J, Hughes SL, et al. B.A.I.L.A. - a Latin dance randomized controlled trial for older Spanish-speaking Latinos: rationale, design, and methods[J]. Contemp Clin Trials. 2014. 38(2): 397-408. 
[2] Năstase. Theoretical design definition of dance sport[J]. Procedia - Social and Behavioral Sciences. 2012. 51: 888-890.

[3] Bronner S. Differences in segmental coordination and postural control in a multi-joint dance movement: développé arabesque[J]. Dance Medicine \& Science. 2012. 16(1): 26-35.

[4] Philippe Perrin DD, Francine Hugel CP. Judo, better than dance, develops sensorimotor adaptabilities[J]. Gait Posture. 2002. 15(2): 187-194.

[6] Wong AM, Chou SW, Huang SC, et al. Does different exercise have the same effect of health promotion for the elderly? Comparison of training-specific effect of Tai Chi and swimming on motor control[J]. Arch Gerontol Geriatr. 2011. 53(2): 133-137.

[7] Fong SM, Ng GY. The effects on sensorimotor performance and balance with Tai Chi training[J]. Arch Phys Med Rehabil. 2006. 87(1): 82-87.

[8] Alfieri FM, Riberto M, Gatz LS, Ribeiro CP, Lopes JA, Battistella LR. Comparison of multisensory and strength training for postural control in the elderly[J]. Clin Interv Aging. 2012. 7: 119-125.

[9] Todd S. Ellenbecker GJD, Jake B. Proprioception and Neuromuscular Control[M]. 2012. 524-547.

[10] Brent LA RJS. Examination of balance measures produced by the biodex stability system[J]. J Athl Train. 1998. 33: 323-327.

[11] Glave AP DJJ, Weatherwax J BSJ, Fiaud V. Testing Postural Stability: Are the Star Excursion Balance Test and Biodex Balance System Limits of Stability Tests Consistent?[J]. Gait Posture. 2016. 43: 225-227.

[12] Sibley KM SSE, Inness EL SNM, Jaglal SB. Balance assessment practices and use of standardized balance measures among Ontario physical therapists[J]. Phys Ther. 2011. 91(11): 1583-1591.

[13] Annelies G. Maenhou TP, Martine De Muynck LFDW, Ann MC. The impact of rotator cuff tendinopathy on proprioception, measuring force sensation[J]. J Shoulder Elbow Surg. 2012. 21: 1080-1086.

(C) 2017 by the authors. Licensee Preprints, Basel, Switzerland. This article is an open access article distributed under the terms and conditions of the Creative Commons by Attribution (CC-BY) license (http://creativecommons.org/licenses/by/4.0/). 